\title{
Magnifying the Early Episodes of Star Formation: Super Star Clusters at Cosmological Distances
}

\author{
E. Vanzella ${ }^{1}$, M. Castellano ${ }^{2}$, M. Meneghetti ${ }^{1}$, A. Mercurio ${ }^{3}$, G. B. Caminha ${ }^{1,4}$, G. Cupani ${ }^{5}$, F. Calura ${ }^{1}$, L. Christensen ${ }^{6}$, \\ E. Merlin ${ }^{2}$, P. Rosati $^{4}$, M. Gronke ${ }^{7}$, M. Dijkstra ${ }^{7}$, M. Mignoli ${ }^{1}$, R. Gilli ${ }^{1}$, S. De Barros ${ }^{8}$, K. Caputi ${ }^{9}$, C. Grillo ${ }^{6,10}$, \\ I. Balestra ${ }^{11}$, S. Cristiani ${ }^{5}$, M. Nonino ${ }^{5}$, E. Giallongo ${ }^{2}$, A. Grazian ${ }^{2}$, L. Pentericci ${ }^{2}$, A. Fontana ${ }^{2}$, A. Comastri ${ }^{1}$, \\ C. Vignali ${ }^{12,1}$, G. Zamorani ${ }^{1}$, M. Brusa ${ }^{1,12}$, P. Bergamini ${ }^{13}$, and P. Tozzi ${ }^{14}$ \\ ${ }^{1}$ INAF-Osservatorio Astronomico di Bologna, Via Gobetti 93/3, I-40129 Bologna, Italy; eros.vanzella@oabo.inaf.it \\ ${ }^{2}$ INAF-Osservatorio Astronomico di Roma, via Frascati 33, I-00040 Monteporzio, Italy \\ ${ }^{3}$ INAF-Osservatorio Astronomico di Capodimonte, Via Moiariello 16, I-80131 Napoli, Italy \\ ${ }^{4}$ Dipartimento di Fisica e Scienze della Terra, Università di Ferrara, via Saragat 1, I-44122 Ferrara, Italy \\ ${ }^{5}$ INAF-Osservatorio Astronomico di Trieste, via G. B. Tiepolo 11, I-34131, Trieste, Italy \\ ${ }^{6}$ Dark Cosmology Centre, Niels Bohr Institute, University of Copenhagen, Juliane Maries Vej 30, DK-2100 Copenhagen, Denmark \\ ${ }^{7}$ Institute of Theoretical Astrophysics, University of Oslo, Postboks 1029 Blindern, NO-0315 Oslo, Norway \\ ${ }^{8}$ Observatoire de Genève, Université de Genève, $51 \mathrm{Ch}$. des Maillettes, 1290, Versoix, Switzerland \\ ${ }^{9}$ Kapteyn Astronomical Institute, University of Groningen, Postbus 800, $9700 \mathrm{AV}$, Groningen, The Netherlands \\ ${ }^{10}$ Dipartimento di Fisica, Università degli Studi di Milano, via Celoria 16, I-20133 Milano, Italy \\ ${ }^{11}$ University Observatory Munich, Scheinerstrasse 1, D-819 München, Germany \\ ${ }^{13}$ Dipartimento di Fisica e Astronomia "G. Galilei," Università di Padova, Vicolo dell'Osservatorio 3, I-35122, Italy \\ ${ }^{14}$ INAF-Osservatorio Astrofisico di Arcetri, Largo E. Fermi, I-50125, Firenze, Italy \\ Received 2017 March 2; revised 2017 May 18; accepted 2017 May 19; published 2017 June 13
}

\begin{abstract}
We study the spectrophotometric properties of a highly magnified ( $\mu \simeq 40-70)$ pair of stellar systems identified at $z=3.2222$ behind the Hubble Frontier Field galaxy cluster MACS J0416. Five multiple images (out of six) have been spectroscopically confirmed by means of VLT/MUSE and VLT/X-Shooter observations. Each image includes two faint $\left(m_{U V} \simeq 30.6\right)$, young $(\lesssim 100 \quad \mathrm{Myr})$, low-mass $\left(<10^{7} M_{\odot}\right)$, low-metallicity $(12+\log (\mathrm{O} / \mathrm{H}) \simeq 7.7$, or $1 / 10$ solar $)$, and compact (30 pc effective radius) stellar systems separated by $\simeq 300$ pc after correcting for lensing amplification. We measured several rest-frame ultraviolet and optical narrow $\left(\sigma_{v} \lesssim 25 \mathrm{~km} \mathrm{~s}^{-1}\right)$ high-ionization lines. These features may be the signature of very hot $(T>50,000 \mathrm{~K})$ stars within dense stellar clusters, whose dynamical mass is likely dominated by the stellar component. Remarkably, the ultraviolet metal lines are not accompanied by $\operatorname{Ly} \alpha$ emission (e.g., C IV $/ \operatorname{Ly} \alpha>15$ ), despite the fact that the Ly $\alpha$ line flux is expected to be 150 times brighter (inferred from the $\mathrm{H} \beta$ flux). A spatially offset, strongly magnified $(\mu>50)$ Ly $\alpha$ emission with a spatial extent $\lesssim 7.6 \mathrm{kpc}^{2}$ is instead identified $2 \mathrm{kpc}$ away from the system. The origin of such a faint emission could be the result of fluorescent Ly $\alpha$ induced by a transverse leakage of ionizing radiation emerging from the stellar systems and/or may be associated with an underlying and barely detected object (with $m_{U V}>34$ de-lensed). This is the first confirmed metal-line emitter at such low-luminosity and redshift without Ly $\alpha$ emission - suggesting that, at least in some cases, a non-uniform covering factor of the neutral gas might hamper the Ly $\alpha$ detection.
\end{abstract}

Key words: cosmology: observations - galaxies: formation - galaxies: high-redshift

\section{Introduction}

Investigation of the ionizing properties of young, low-mass, star-forming systems caught at $z \simeq 3$ (i.e., nearly 1 Gyr after reionization ended and the possible analogy with similar systems identified during reionization $(z>6)$ ) currently represents a strategic line of research, especially at poorly explored, low-luminosity regimes (e.g., Amorín et al. 2017). The detection of nebular high-ionization emission lines has underscored the considerable contribution of hot and massive stars to the transparency of the medium in low-luminosity ( $\left.L \ll L^{*}\right)$ systems (Vanzella et al. 2016a), as expected in scenarios in which stellar winds and supernova explosions blow cavities in the interstellar medium (e.g., Jaskot \& Oey 2013; Micheva et al. 2017). In fact, the rest-frame

\footnotetext{
* Based on observations collected at the European Southern Observatory for Astronomical research in the southern hemisphere, under ESO programmes P095.A-0840, P095.A-0653, and P186.A-0798.
}

ultraviolet and optical line ratios observed in $z \simeq 0, z=2-3$ and $z>6$ galaxies suggest a possible evolution of the ionizing radiation field, such that the presence of hot stars seems more common at high redshift, with blackbody mean effective temperatures on the order of 50.000-60.000 K at $z=2-3$ (Fosbury et al. 2003; Steidel et al. 2014; Holden et al. 2016), or even hotter at $z>6$ (Stark et al. 2015; Mainali et al. 2016).

Moreover, in very recent times, strong gravitational lensing has often been used to investigate the physical properties of such intrinsically faint, low-mass systems. Its use is now reaching its maturity thanks to dedicated deep imaging studies in lensed fields (e.g., the Hubble Frontier Fields program, HFF hereafter, Koekemoer et al. 2014; Lotz et al. 2014, 2016), as performed on $3<z<8$ dropout galaxies (e.g., Bouwens et al. 2016) and spectroscopic surveys (e.g., Stark et al. 2014; Vanzella et al. 2014, 2016a, 2017b; Caminha et al. 2016c; Mainali et al. 2016; Karman et al. 2017; Stark et al. 2017). In 
this regard, the exceptional line flux sensitivity of the integral field spectrograph MUSE mounted on the VLT (Bacon et al. 2010) ${ }^{15}$ has driven considerable progress. In fact, MUSE allows the identification and characterization of extremely faint (and small) line emitters at $3<z<6.6$, without the need of specific pre-selection of the targets in a relatively large field of view $\left(1^{\prime} \times 1^{\prime}\right)$, aptly matching strongly magnified regions of the sky. In these investigations, strong lensing turned out to be essential for two reasons: (1) before the completion of the Extremely Large Telescope (ELT), it is not possible to spatially resolve sources with effective radii lower than $150 \mathrm{pc}(<20$ mas) at $z>3$ in nonlensed fields. Conversely, galaxy cluster lensing has already enabled us to perform light profile fitting of dwarf and super star-clusters with radii $15-100$ pc at $3<z<6.5$ (e.g., Bouwens et al. 2016; Vanzella et al. 2016a, 2017b); (2) the increased $\mathrm{S} / \mathrm{N}$ of the SEDs and emission lines allows us to access the physical properties down to (intrinsic) magnitudes $m>29$ and line fluxes of $>5-10 \times 10^{-20} \mathrm{cgs}$.

MUSE observations on HFFs are leading us to the construction of a reference sample, at $z<4$, of faint, starforming compact analogs of the systems active during cosmic reionization. Such a task is particularly important, given the imminent launch of the James Webb Space Telescope (JWST), whose primary aim is to capture the first episodes of star formation at $z>6$.

At $z \simeq 2-3$, the optical rest-frame is accessible from groundbased spectrographs. A large number of strongly lensed systems have been identified (e.g., Alavi et al. 2016; Caminha et al. 2016a, 2016b, 2016c; Karman et al. 2017), allowing us to spectroscopically probe extremely low-luminosity and smallmass regimes with unprecedented detail (e.g., Christensen et al. 2012; Stark et al. 2014; Vanzella et al. 2016a). Some of these systems have already provided a valuable insight of the physical conditions characterising newborn, highly ionized, dwarf galaxies (e.g., Vanzella et al. 2016a, 2016b) and extremely compact star clusters, presenting features very similar to those expected during the formation of globular clusters (Vanzella et al. 2017b). An intriguing new system of this kind is that discussed here, i.e., a strongly lensed system at $z=3.2222$ that shows high ionization lines detected behind the HFF galaxy cluster MACS J0416 (Caminha et al. 2016c). In addition to its compactness and extreme faintness, the distinguishing feature of this system is the presence of strong ionization lines that contrast with the absence of Ly $\alpha$ emission. These features are very rare among known local and distant star-forming systems (e.g., Stark et al. 2014, 2015; Henry et al. 2015; Mainali et al. 2016; Shibuya et al. 2017; Smit et al. 2017). The intriguing nature of our system and its ionization structure provides us with an insightful example of the geometrical complexity of high-redshift young, ionized systems. The energy and momentum continuously deposited by massive, young stellar associations can lead to puzzling structures such as our object, which also represents a valuable benchmark for interstellar photo-ionization models.

In this work, we use the Hubble Frontier Fields HST-data set $^{16}$, in particular, three optical and four near-infrared bands: F435W, F606W, and F814W (HST/ACS); and F105W, F125W, F140W, and F160W (HST/WFC3), with typical

\footnotetext{
15 http://www.eso.org/sci/facilities/develop/instruments/muse.html

16 http://www.stsci.edu/hst/campaigns/frontier-fields/HST-Survey
}

limiting $\mathrm{AB}$ magnitudes 28.5-29.0 calculated at 5 $\sigma$ depth. In addition to the seven HST bands, the publicly available Hawk-I@VLT Ks band and the Spitzer/IRAC 3.6 and 4.6 $\mu \mathrm{m}$ data have also been included. A description of the data set, the extraction of the PSF-matched photometry, and the construction of the photometric catalog are provided in Castellano et al. (2016) and Merlin et al. (2016).

This paper is organized as follows. In Section 2, a brief description of the multiple-image magnification pattern of our system is presented. Our main results are presented in Section 3, and discussed in Section 4. Finally, some conclusions are drawn in Section 5.

Throughout the paper, we assume a flat cosmology with $\Omega_{M}=0.3, \Omega_{\Lambda}=0.7$, and $H_{0}=70 \mathrm{~km} \mathrm{~s}^{-1} \mathrm{Mpc}^{-1}$.

\section{ID14, a Double-lensed System}

The source ID14 is multiply imaged by the cluster MACS J0416 into three images separated by up to $50^{\prime \prime}$. The location of these three images is given, in the right panel of Figure 1, by the three yellow circles labelled Image1, Image2, and Image3. Image1 happens to be close to a pair of elliptical cluster members, indicated as E1 and E2 in the left panel of Figure 1. These galaxies act as strong lenses, further splitting Image1 into the four images ID14a, ID14b, ID14c, and ID14d. For consistency, in the following, we will refer to Image 2 and Image 3 as ID14e and ID14f, respectively.

This system was first discovered by Zitrin et al. (2013). In each of the images, the source appears to consist of two bright knots. This morphological marker, along with the image geometry, allowed the first association of the images to the same source. The two knots are labelled " 1 " and " 2 " in Figure 1. As discussed in the next section, the images ID14a, b, $\mathrm{c}, \mathrm{d}$ are spectroscopically confirmed. The association of Image 3 with the sixth multiple image of ID14 (namely ID14f) is corroborated by the lens model recently presented by Caminha et al. (2016c), which predicts the presence of an image at this location within $1 \sigma$ uncertainty, i.e., $<1^{\prime \prime}$. In addition, the photometric redshift of ID14f is zphot $=3.32 \pm 0.10$ (see Figure 2), which is fully consistent with the spectroscopic redshift of ID14. ${ }^{17}$

For the analysis discussed in this paper, it is crucial to estimate the magnification of the brightest images of ID14, namely images $a, b$, and $c$. For this goal, the lens model by Caminha et al. (2016c) could, in principle, be used. There are, however, two complications. The first is that these images are located in a region of high magnification. In a collaborative work that involved several groups employing different lens modeling algorithms, among them the one used by Caminha et al. (2016c), Meneghetti et al. (2016) recently showed that the model magnifications in this regime are affected by large uncertainties. For example, at $\mu>10$, the error on the local magnification estimates is $\gtrsim 50 \%$. This is due to the strong magnification gradient near the critical lines. The second complication is that images ID14a, b, and c are the result of a galaxy-galaxy strong lensing event. Meneghetti et al. (2016) also showed that the model uncertainties increase near cluster members.

For these reasons, instead of reading the magnifications off the map derived from the Caminha et al. (2016c) model, we

\footnotetext{
17 The photometric redshift is also reported in Castellano et al. (2016). In particular, an example of the best-fit SED for ID14f is shown at following address http://astrodeep.u-strasbg.fr/ff/?ffid=FF_M0416CL\&id=1141.
} 


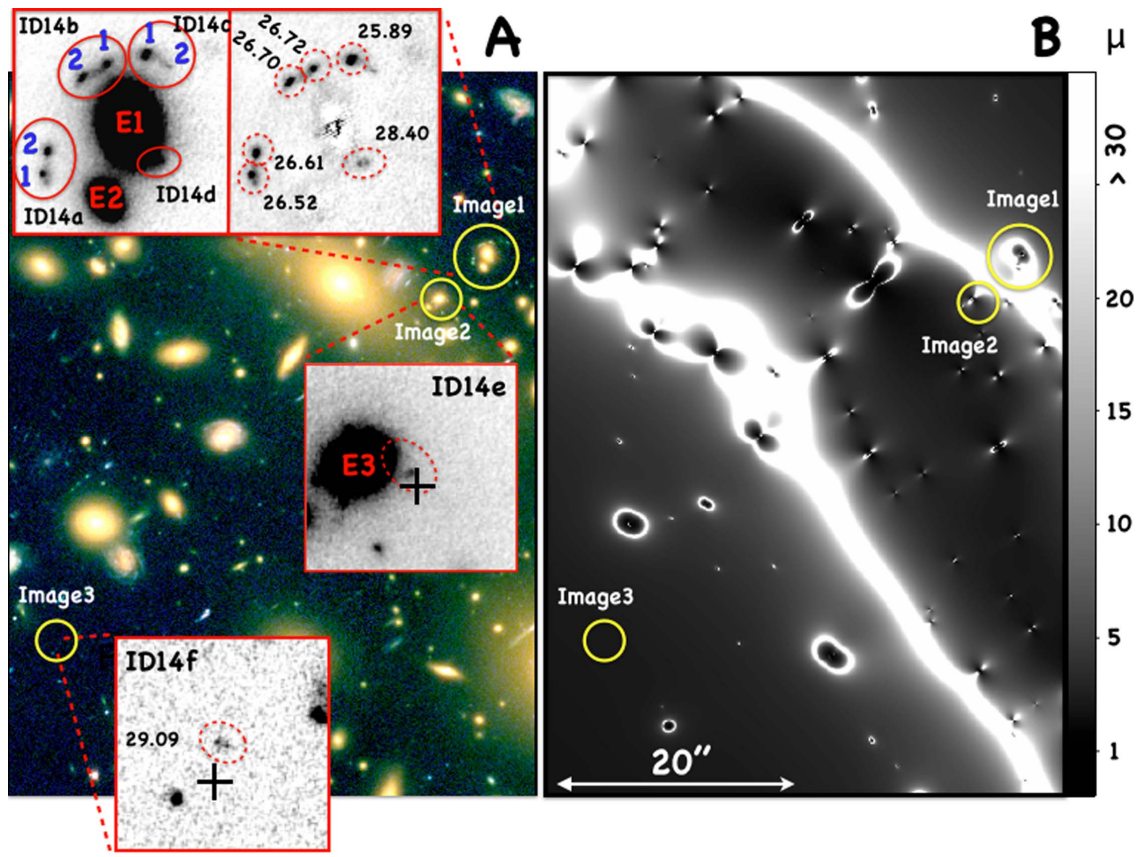

Figure 1. Panel (A): the HST color image of the galaxy cluster MACS J0416, showing the position of the three multiple images expected at the redshift of ID14 generated by the galaxy cluster ("Image1," "Image2," and "Image3"). "Image1" is further magnified by the elliptical galaxy cluster members E1+E2 into four additional multiple images (ID14a, b, c, d). The left and right top insets show the F814W image with and without the subtraction of the galaxies E1+E2. Each image of ID14 contains two further components, "1" and "2." Magnitudes correspond to the average values obtained from all the HST bands (except F435W). The other two multiple images, "Image2" (ID14e) and "Image3" (ID14f), are also shown in the insets (red dotted circles), where the black crosses mark the position predicted by the lens model. All insets have a size of 3"! 3). Panel (B): magnification map ( $\mu$, coded according to the grayscale bar on the right) derived from the lens model by Caminha et al. (2016c): large magnifications are expected close to the critical lines, between "Image1" and "Image2" and between ID14a, b, and c.

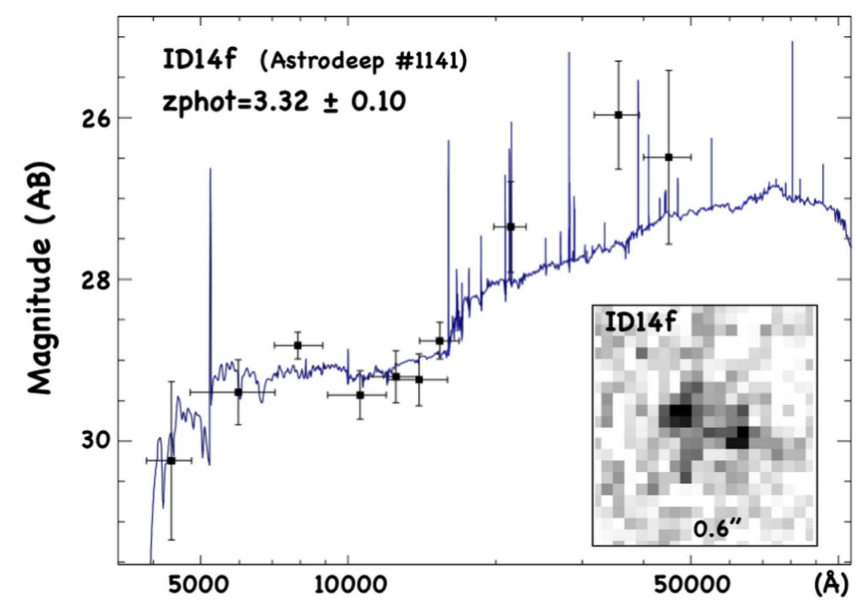

Figure 2. Best-fit SED solution (blue template) compared to the photometry (black points) for image ID14f. The photometric redshift, with its $1 \sigma$ uncertainty, is reported (see also Castellano et al. 2016). The inset shows the zoomed ID14f image in the F814W band. The photometric black points includes both components " $1,2$. ."

opted to derive the magnifications of ID14a, b, and c using the same method employed in Vanzella et al. (2016a, 2017b). Meneghetti et al. (2016) showed that magnification estimates are significantly more robust if $\mu<5$. Using the lens model by Caminha et al. (2016c), we estimate that the magnification of ID14f is $\mu=2.0 \pm 0.1$. This estimate is consistent with others based on different models of MACS J0416, which we obtained from the Hubble Frontier Field Magnification calculator. If we assume that the model magnification of this image is secure, we can then derive the magnifications of the other images by means of the measured flux ratios.
ID14f is identified with Astrodeep object \#1411 (ID1411) (Castellano et al. 2016; Merlin et al. 2016). It is detected in the F814W, F105W, F125W, F140W, and F160W bands, with an average magnitude of $29.09 \pm 0.15$ resulting from the sum of both components " 1 " and " 2 ." We computed multi-band, PSFmatched photometry of images ID14a, b, and c as described in Merlin et al. (2016), but using the F814W band as detection image instead of F160W. HST photometry has been measured from 30 mas pixel-scale images, after subtracting the elliptical galaxies E1+E2 with Galfit (Peng et al. 2010). T-PHOT v2.0 (Merlin et al. 2016) has been used to measure $K$ and IRAC photometry. An example of subtracted ellipticals in the F814W-band is shown in Figure 1 (top-left inset), where also the average magnitudes (over the five HST bands) are quoted for each component. For example, image ID14a is composed of two components " 1 " and " 2 " with magnitudes 26.52 and 26.61, respectively. The total magnitude, combining the two components, is 25.81 . The total magnification for ID14a is therefore $\mu($ ID14a $)=\mu($ ID14f $) \times$ $($ flux[ID14a] $/$ flux[ID14f] $)=41 \pm 7$. Similarly, $\mu($ ID14b) $=$ $36 \pm 7$ and ID14c.1 (the component " 1 " only) is magnified by a factor $76 \pm 10$. The errors estimated with this method are $<20 \%$, despite the large magnification regime. The de-lensed magnitude of $\operatorname{ID} 14 \mathrm{f}(1+2)$ is 29.84 . Assuming a flux ratio of $\sim 1$ between " 1 " and " 2 " (as observed in images ID14a and b), the intrinsic magnitude of each component is $\simeq 30.60$.

It is worth comparing the above values with those inferred directly from the Caminha et al. model and other independent models. The best estimates and the $1 \sigma$ statistical errors from Caminha et al. for images ID14a(1) and ID14a(2) are $\mu=33_{-12}^{+38}$ and $\mu=23_{-6}^{+15}$, respectively $\left(\mu=29_{-17}^{+64}\right.$ and $\mu=22_{-11}^{+34}$ for ID14b1 and 2). They change significantly if different and 


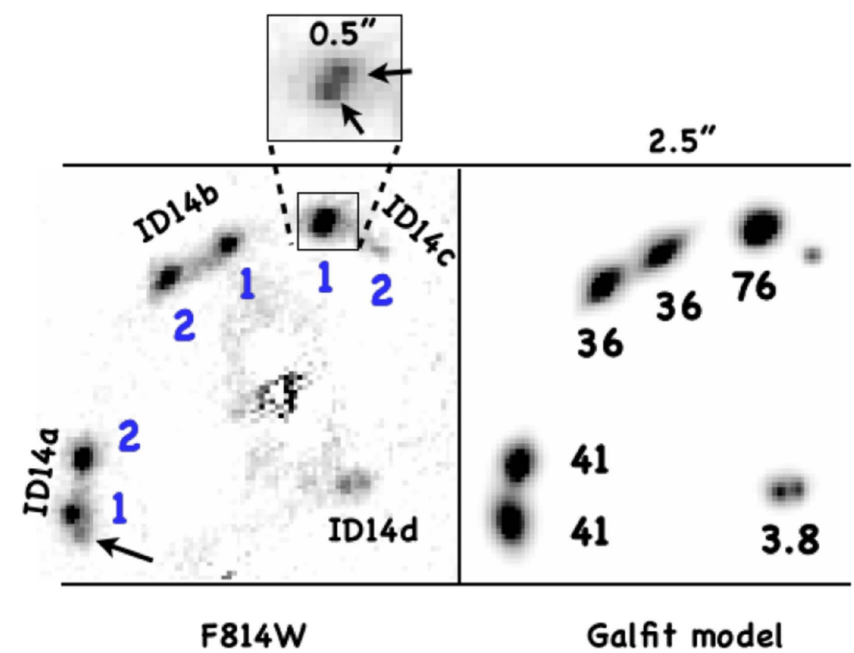

Figure 3. Left panel: the multiple images ID14a, b, c, and d are shown in the F814W band. The morphological details of images ID14a and ID14c are indicated by the black arrows and zoomed inset (for ID14c). The corresponding Galfit models of each component are shown on the right side (the numbers indicate the magnification, as derived in Section 2).

independent lens models are applied. ${ }^{18}$ In particular, the magnifications of images ID14a(1) and ID14a(2) inferred from eleven lens models span the interval 8-110 with medians $\mu \simeq 30-40$. Also the reported $1 \sigma$ uncertainties in each model are larger than $50 \%$. The same happens also for the other strongly magnified images, ID14b and ID14c. The scatter among the various model predictions is on the order of the predicted magnifications, suggesting that the systematic errors dominate the uncertainties, especially in this complex double-lensed object (see Meneghetti et al. 2016 for a detailed discussion). Therefore, the method used in this work significantly decreases the uncertainties of the amplification factors, and consequently limits the error budget on the intrinsic physical properties discussed in the next section.

\section{Characterizing System ID14}

The main goal of our investigation is to estimate the size and the age of our system, as well as to assess the nature of the radiation field originated by its two close, compact sources. The gas phase metallicity is also estimated, as well as the dynamical mass, useful to constrain the baryon content of ID14.

\subsection{Sizes of the Components "1" and " 2 "}

Galfit fitting (Peng et al. 2010) has been performed on both components " 1,2 " of images ID14a, b, and c (following the methodology described in Vanzella et al. 2017b) after the elliptical galaxies, E1 and E2, have been subtracted (Figure 1, top-left). All the multiple images are very nucleated and tangentially elongated along the arc shape showing an average effective radius $\left\langle R_{e}\right\rangle=1.5 \pm 0.3$ pix and an axis ratio $(q=b / a)\langle q\rangle=0.3 \pm 0.2$ (an example of Galfit modeling is shown in Figure 3). The circularized radius $R_{c}\left(=R_{e} \times q^{0.5}\right)$ is 0.8 pix ( 1 pix $=0$ ". 03 ) corresponding to a de-lensed size of $\simeq 30$ pc at $z=3.222$, adopting $\mu=40$, (or sizes lower than 50 pc, if we increase $R_{e}$ to 2 pix and decrease $\mu$ to 30 ). The

\footnotetext{
${ }^{18}$ We used the Hubble Frontier Field Magnification calculator, https:// archive.stsci.edu/prepds/frontier/lensmodels/.
}

two components " 1 " and " 2 " are separated by 300 pc on the source plane.

Additional details on the observed multiple images are highlighted in Figure 3 (see ID14c), but we will not discuss them further in this work. We stress that these additional features are at least two magnitudes fainter than the main components (or constitute sub-structures of the components themselves). Given the large magnification, they would have a de-lensed ultraviolet magnitude fainter than 31 and de-lensed sizes possibly consistent with forming star-clusters on parsecscale. These additional features might also be the result of the extremely large magnification gradient characterizing this field.

\subsection{Spectral Features}

The 2h VLT/MUSE (ID 094.A-0115B, PI: J. Richard) and 2h VLT/X-Shooter (ID 098.A-0665B, PI: E. Vanzella) spectra of images ID14 are shown in Figures 4 and 5, obtained with good seeing conditions, $0 . / 7$ and $0 . / 5$, respectively. The apertures over which the spectra have been extracted are also shown in the same figure. Data reduction for X-Shooter and MUSE have been performed as described in Vanzella et al. (2016a) and Caminha et al. (2016c), respectively.

The most prominent line in the MUSE spectrum is the C IV $\lambda 1548$ component of the C IV doublet. We use this line to measure the redshift of the multiple images contained in the data-cube (only ID14f is not included in the MUSE field of view). A continuum-subtracted data-cube has been computed by averaging the signal in a central window of three spectral elements (one element corresponds to $1.25 \AA$ ) and subtracting the average signal from two adjacent windows of 10 spectral elements each (see also Vanzella et al. 2017a). Figure 6 (left panel) shows the result. The C IV $\lambda 1548$ line is detected for all images ID14a, b, c, d, and e with $\mathrm{S} / \mathrm{N}>5$.

Thanks to lensing magnification, the photometric properties and sizes of components " 1 " and " 2 " can be studied individually. On the other hand, the ultraviolet and optical spectra are seeing-limited and contain the contribution of both components. It is worth noting that the C IV emission (especially for ID14a) shows an elongated shape that follows the spatial orientation of components " 1 " and " 2 ." This suggests that both components contribute to the line emission, and to the ultraviolet metal lines in general. MUSE equipped with the adaptive optics module may allow these spectral features to be spatially resolved.

We exploited the MUSE data-cube by choosing a free-form aperture encompassing the three images ID14a, b, and $\mathrm{c}$ (increasing the $\mathrm{S} / \mathrm{N}$ ); on the other hand, the $\mathrm{X}$-Shooter slit captures the image ID14b and one component (" 1 ") of the image ID14c (that being the component " 2 ," less magnified; see Figure 5, top left). Line fluxes, signal-to-noise, velocity dispersions, rest-frame equivalent widths (EWs) and redshifts are reported in Table 1. High ionization lines C IV $\lambda 1548,1550$, He II $\lambda 1640$, O III] $\lambda 1661$, and 1666, as well as C III] $\lambda \lambda 1907$ and 1909 have been detected, with $\mathrm{S} / \mathrm{N}$ ratios ranging between 2 and 20 and the X-Shooter lines, $\mathrm{H} \beta$, [O III] $\lambda \lambda 4959$, and 5007, with $\mathrm{S} / \mathrm{N}=4-31$. High ionization lines have also been confirmed with $\mathrm{X}$-Shooter (with $R=7400$ ), although only $\mathrm{C}$ IV $\lambda 1548$ and $\mathrm{O}$ III] $\lambda 1666$ have $\mathrm{S} / \mathrm{N} \gtrsim 3$ (see Figure 5). Remarkably, no Ly $\alpha$ emission has been detected $(\mathrm{S} / \mathrm{N}<3)$ in the same spatial region where both the metal lines and stellar continuum arise. We discuss this in Section 4.3. The observed line fluxes derived from the aforementioned apertures are 

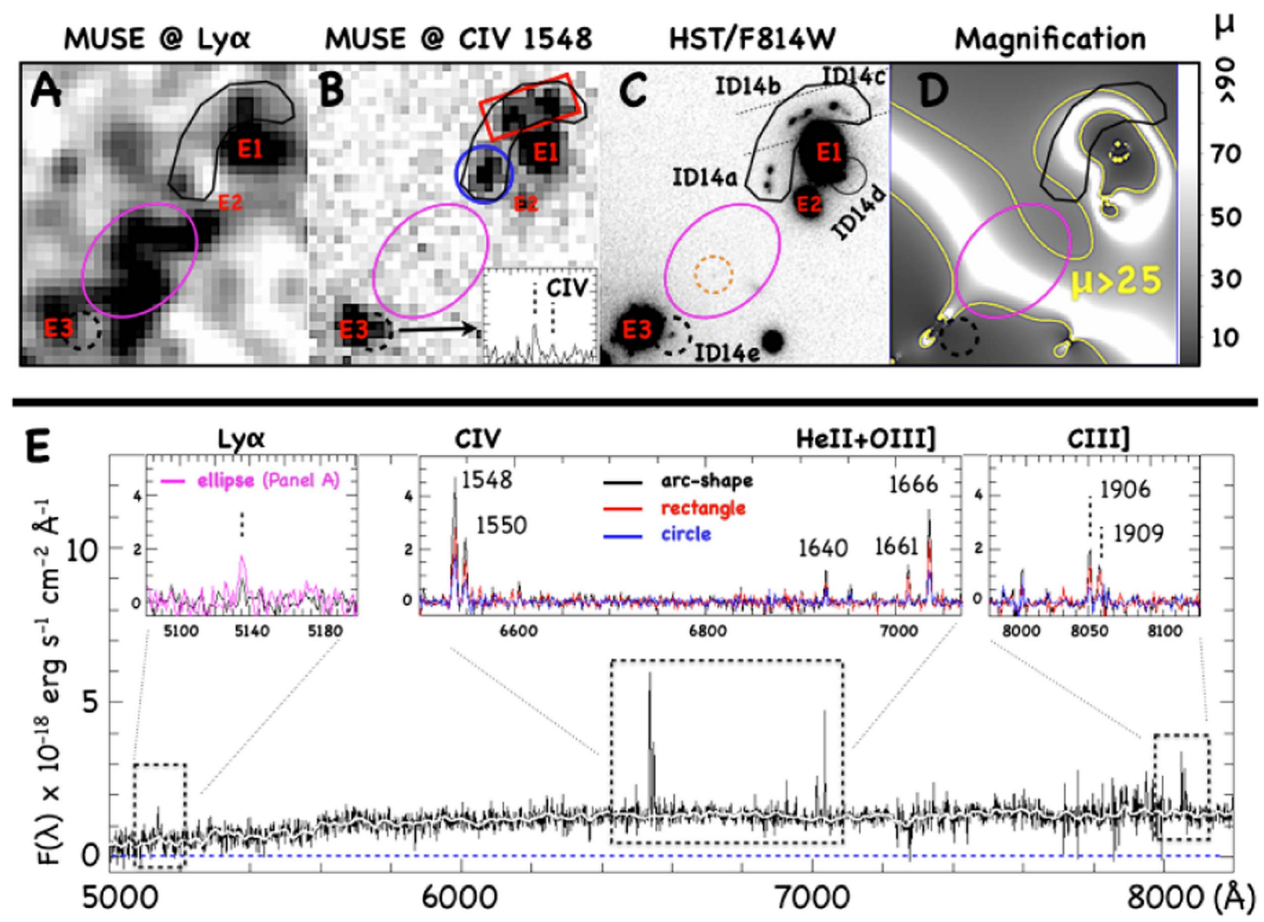

Figure 4. Panoramic view of the MUSE spectra. Top panels: the zoomed region centered on ID14a, b, c, d, and e is shown (6". 8 wide). From left to right, we show the MUSE images at the Ly $\alpha$ (panel (A)), at $1548 \AA$ (panel (B)), in the HST/F814W band (panel (C)), and the gray-coded magnification map (panel (D)), in which the yellow contours enclose the region with $\mu>25$. The arc-shaped black line marks the aperture used to extract the MUSE spectrum as the sum of images ID14a, $\mathrm{b}$, and c. The black dashed circle identifies the image ID14e and the magenta ellipse the diffuse Ly $\alpha$ emission at the same redshift as ID14 on top of the critical line of the galaxy cluster. Panel (B) shows the rectangular red aperture with the same width and position angle of the X-Shooter slit (also shown in panel (C), top-right, with black dotted thin lines). The orange dotted circle in panel (C) marks the position of a possible counterpart of the diffuse Ly $\alpha$ emission (magenta ellipse). The blue circle ( 1 !! 2 diameter) marks the aperture used to derive the spectrum for image ID14a only. Panel (E), shows the MUSE spectrum of ID14a, b, and c (black line) extracted using the arc-shaped aperture. The continuum of the elliptical galaxies E1+E2 has been subtracted (white thick line) and the high ionization lines are shown in the corresponding insets. The colors of the spectra in the insets corresponds to the colors of the apertures shown in panel (B). The flux scale of all images is $F(\lambda)\left[10^{-18} \mathrm{erg} \mathrm{s}^{-1} \mathrm{~cm}^{-2} \AA^{-1}\right]$.

reported in column \#2 of Table 1 . Because they include different portions of the multiple images, we rescaled properly the fluxes in column \#2 to obtain the values associated to the single object ID14 ("1, 2"). The corrected fluxes are reported in column \#3, $F_{\text {corr }}$, obtained by dividing the measured fluxes reported in column \#2 by 2.5 and 1.5 in the case of MUSE and $\mathrm{X}$-Shooter observations, respectively. The two rescaling factors have been derived from specific apertures defined in the MUSE data-cube: a circular aperture centered on ID14a and a rectangular aperture mimicking the X-Shooter slit (see Figure 4, panel (B)). The EWs of the lines have been derived using $F_{\text {corr }}$ and the inferred continuum magnitude from the SED-fitting of both components "1" and "2" (see Section 3.3). Typical errors mainly reflect uncertainty in the line flux, as the underlying images are well-detected in the deep HFF images. Conservatively, even including the possible systematic errors (due to the subtraction of E1+E2) the final error on the EWs is not larger than $50 \%$.

Beside the presence of ultraviolet metal lines, also the prominent $[\mathrm{O} \mathrm{III}] \lambda \lambda 4959$ and 5007 line emission, the ratios $[\mathrm{O}$ III $] \lambda \lambda 4959,5007 /[\mathrm{O}$ II $] \lambda 3727$, and $3729>15$, as well as $[\mathrm{O}$ III $] \lambda \lambda 4959$ and $5007 / \mathrm{H} \beta>10$ suggest a highly ionized medium and a possibly large Ly $\alpha$ escape fraction (Erb et al. 2016; Henry et al. 2015). Despite that, the Ly $\alpha$ emission in ID14 is deficient (see Section 4), independently from the adopted aperture shape.

\subsection{Physical Properties}

SED fitting has been performed using BC03 templates (Bruzual \& Charlot 2003) on each component " 1 " and "2," for images ID14a and b, as well as component " 1 " of ID14c (see Castellano et al. 2016), after subtracting the elliptical galaxies E1+E2 in all the HST images and using the T-PHOT v2.0 algorithm, Merlin et al. (2016) (see Section 2). The subtraction of E1+E2, however, may introduce non-negligible color trends, especially in the nearinfrared bands. Such residuals may introduce systematics in the inferred physical quantities. In order to add constraints to our photometric analysis, we also estimate the dynamical mass and the gas phase metallicity. The inferred dynamical mass from the $\sigma_{v}([\mathrm{O}$ III $] \lambda 5007) \lesssim 20 \mathrm{~km} \mathrm{~s}^{-1}$ velocity dispersion is $M_{\text {dyn }}<$ $2 \times 10^{7} M_{\odot}$, where we assume the [O III] $\lambda 5007$ line width is associated to both components "1" and "2" of $30 \mathrm{pc}$ radius each (see discussion in Erb et al. 2014; Rhoads et al. 2014; Vanzella et al. 2017b). The metallicity is computed from the highionization and optical rest-frame line ratios (Pilyugin et al. 2006; Pérez-Montero \& Amorín 2017). The electron temperature $T_{e}=19,000 \pm 1000 \mathrm{~K}$ is derived from the observed $\mathrm{O}$ III] $\lambda 1661$ and $1666 /[\mathrm{O}$ III] $] \lambda 5007$ ratio. Our estimate of $T_{e}$ is not sensitive on the assumed electron density in the range $n_{e}=10-10,000 \mathrm{~cm}^{-3}$. We obtain $12+\log (\mathrm{O} / \mathrm{H}) \simeq 7.7 \pm 0.2$, which corresponds to about $1 / 10$ solar. We performed the SED fitting by fixing metallicity to the measured value and considering only those solutions producing stellar masses not exceeding 10 times the 

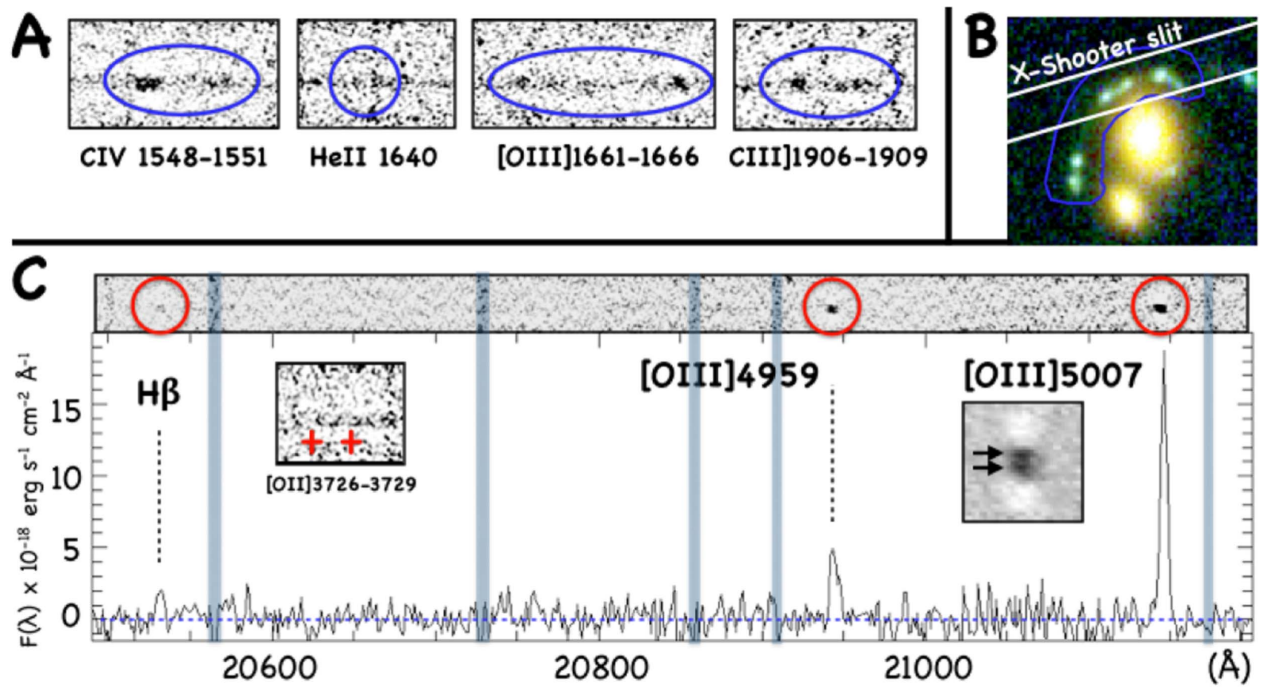

Figure 5. X-Shooter spectra in the VIS and NIR arms are shown for the relevant emission lines. In panel (A), the two-dimensional zoomed spectra of the UV metal high-ionization lines are shown (the faint continuum comes from the foreground elliptical galaxy). In panel (B) the color image of ID14a, b, c, and d is shown, with the MUSE aperture used to extract the spectra (blue curve) and the orientation and width $\left(0{ }^{\prime \prime} 9\right)$ of the X-Shooter slit (white lines). The size of the image is $3^{\prime \prime}$ on a side. The one- and two-dimensional $\mathrm{H} \beta$ and $[\mathrm{O}$ III] $] \lambda \lambda 4959,5007$ lines observed at $\simeq 2 \mu \mathrm{m}$ are shown in panel (C). The left inset shows the two-dimensional spectrum centered at the position of the expected [O II] $\lambda 3727,3729$ doublet. The right inset is a zoomed image of [O III] $\lambda 5007$, in which ID14b and ID14c are spatially resolved (the arrows mark their position).

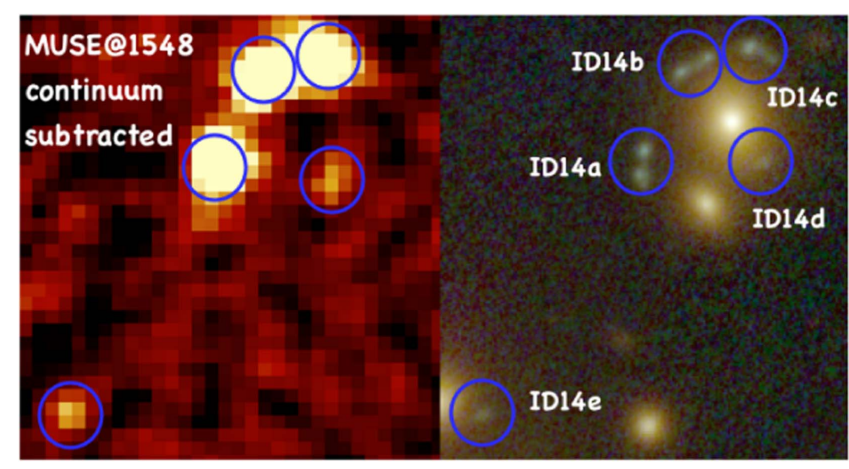

Figure 6. The continuum-subtracted data-cube at the C IV $\lambda 1548$ wavelength position is shown on the left panel (6". 5 wide). The blue circles mark the multiple images of ID14 detected on the HST color image, reported in the right panel. In particular, faint line emissions arise at the position of multiple images ID14d and ID14e.

estimated dynamical mass (the factor ten includes the uncertainties in the $M_{\text {dyn }}$ estimate). In general, the inferred quantities agree among the multiple images of the same component.

In particular, we focus on images ID14a.1 and ID14a.2, which are the least contaminated by the elliptical galaxies $\mathrm{E} 1+\mathrm{E} 2$. The best-fit yields stellar masses $\lesssim 10^{7} M_{\odot}$, ages $\lesssim 100 \mathrm{Myr}, \mathrm{SFRs} \simeq 0.1 M_{\odot} \mathrm{yr}^{-1}$ and $E(B-V)<0.1$. Table 2 summarizes the results and Figure 7 shows the best-fit solutions. It is worth noting that the $\simeq 1 \mathrm{mag}$ excess in the $\mathrm{K}$-band is fully consistent with the observed [O III] $\lambda \lambda 4959$, 5007 flux. In particular, the measured equivalent width of EW $([\mathrm{O}$ III $] \lambda 5007) \simeq 1200 \AA$ rest-frame, the inferred metallicity, young ages, and low stellar mass place this object in the category of the so-called "Green Pea" (GP) sources, although the stellar mass and size are, in our case, an order of magnitude lower than those typically reported (Cardamone et al. 2009; Amorín et al. 2015, 2017; Henry et al. 2015). In particular, Henry et al. (2015) selected a sample of 10 Green Pea sources $(z \sim 0.2)$ on the basis of high EW optical emission lines, and
Table 1

Observed Spectral Lines

\begin{tabular}{|c|c|c|c|}
\hline line $/ \lambda_{\text {vacuum }}$ & {$\left[F_{\mathrm{obs}}\right]\left(\frac{\mathrm{S}}{\mathrm{N}}\right)\left(\sigma_{v}\right)$} & {$\left[F_{\text {corr }}\right](\mathrm{EW})$} & Redshift \\
\hline Ly $\alpha 1215.69$ & {$[0.24](2.5)(-)$} & {$[0.09](\simeq 1.1)$} & {$[\mathrm{M}](3.226)$} \\
\hline$[\mathrm{C}$ IV $] \lambda 1548.20$ & {$[2.18](20.0)(\lesssim 64)$} & {$[0.87](16.9)$} & [M] 3.222 \\
\hline$[\mathrm{C}$ IV $] \lambda 1550.78$ & {$[1.20](11.0)(\lesssim 64)$} & {$[0.48](9.3)$} & {$[\mathrm{M}] 3.223$} \\
\hline He II $\lambda 1640.42$ & {$[0.33](4.8)(<38)$} & {$[0.13](2.8)$} & [M] 3.223 \\
\hline O III] $\lambda 1660.81$ & {$[0.48](5.4)(<42)$} & {$[0.19](4.3)$} & [M] 3.222 \\
\hline O III] $\lambda 1666.15$ & {$[1.02](11.7)(<42)$} & {$[0.41](9.2)$} & [M] 3.222 \\
\hline [C III] $] \lambda 1906.68$ & {$[0.55](6.2)(<42)$} & {$[0.22](6.5)$} & [M] 3.223 \\
\hline C III] $\lambda 1908.73$ & {$[0.45](5.1)(<42)$} & {$[0.18](5.3)$} & [M] 3.222 \\
\hline [O II] $] \lambda 3727.09$ & {$[<0.28](1)$} & $\ldots$ & {$[\mathrm{X}] \cdots$} \\
\hline$[\mathrm{O}$ II $] \lambda 3729.88$ & {$[<0.28](1)$} & $\cdots$ & {$[\mathrm{X}] \cdots$} \\
\hline [O II] $] \lambda 4364.44$ & {$[<0.28](1)$} & $\ldots$ & {$[\mathrm{X}] \cdots$} \\
\hline $\mathrm{H} \beta \lambda 4862.69$ & {$[1.06](3.7)(\cdots)$} & {$[0.71](136)$} & {$[\mathrm{X}](3.2222)$} \\
\hline [O III] $\lambda 4960.30$ & {$[2.76](9.6)(<23)$} & {$[1.84](367)$} & [X] 3.2222 \\
\hline [O III] $\lambda 5008.24$ & {$[8.92](31.5)(<18)$} & {$[5.95](1209)$} & [X] 3.2222 \\
\hline Ly $\alpha 1215.69$ & {$[0.85](7.4)(<40)$} & $\ldots$ & [M] 3.223 \\
\hline
\end{tabular}

Note. Column \#1: the observed emission lines and their rest-frame wavelengths. Column \#2: observed fluxes (in units of $10^{-17} \mathrm{erg} \mathrm{s}^{-1} \mathrm{~cm}^{-2}$ ), $\mathrm{S} / \mathrm{N}$ and $\sigma_{v}$ (instrument-corrected velocity dispersion in $\mathrm{km} \mathrm{s}^{-1}$ ) for ID14 extracted from the apertures that include the multiple images. Flux limits are reported at $1 \sigma$. Column \#3: Fluxes associated to the object ID14 (components "1" and "2") used to derive flux ratios and intrinsic quantities ( $F_{\text {corr }}$; see the text for details). The rest-frame EW ( $\AA$ ), derived adopting the SED-fitted continuum of "1"+"2" $(m \simeq 25.80)$, is also reported. The de-lensed fluxes can be obtained by dividing these values by $\mu=40$. In column \#4, the MUSE and $\mathrm{X}$-Shooter redshifts are indicated with $[\mathrm{M}]$ and $[\mathrm{X}]$, respectively (redshifts in parenthesis are uncertain). The $\sigma_{v}$ of the lines with $\mathrm{S} / \mathrm{N}>3$ are estimated from the higher-resolution X-Shooter spectrum when possible, or from MUSE otherwise. The Ly $\alpha$ flux calculated within the magenta ellipse (whose area is 6.7 sq. arcsec), as shown in Figure 4, is reported in the last row.

found strong $\operatorname{Ly} \alpha$ emission in all of them. Remarkably, although ID14 shows many properties in common with the GPs sources, the Ly $\alpha$ is deficient (see next section). 
Table 2

Properties of Images ID14a and ID14f

\begin{tabular}{lllc}
\hline \hline & ID14a(1) & ID14a(2) & ID14f(1+2) \\
\hline$M_{\text {stellar }\left[10^{6} M_{\odot}\right]}$ & $2.5-9.8$ & $2.0-7.6$ & $\ldots$ \\
SFR $\left[M_{\odot} \mathrm{yr}^{-1}\right]$ & $0.10-0.15$ & $0.10-0.17$ & $\ldots$ \\
Age $[\mathrm{Myr}]$ & $16-100$ & $13-80$ & $\ldots$ \\
$E(B-V)$ & $\simeq 0.06$ & $\simeq 0.06$ & $\ldots$ \\
$R_{c}(\mathrm{UV})[\mathrm{pc}]$ & $30 \pm 11$ & $30 \pm 11$ & $\ldots$ \\
$m_{U V}(\mathrm{obs})$ & $26.52 \pm 0.03$ & $26.61 \pm 0.03$ & $29.09 \pm 0.15$ \\
$m_{U V}(\mathrm{int})$ & $30.55 \pm 0.19$ & $30.64 \pm 0.19$ & $29.84 \pm 0.16$ \\
$M(1500 \AA)(\mathrm{int})$ & -15.12 & -15.03 & -15.85 \\
$\beta_{U V}$ & $-1.98 \pm 0.20$ & $-1.98 \pm 0.20$ & $-2.0 \pm 0.5$ \\
$\mu$ & $40 \pm 7$ & $41 \pm 7$ & $2.0 \pm 0.1$ \\
\hline
\end{tabular}

Note. De-lensed physical properties are derived from SED-fitting for the single components " 1 " and " 2 " of image ID14a, and the combination " $1+2$ " of ID14f by adopting the measured metallicity (see text). The $68 \%$ central intervals are reported for the stellar mass, star-formation rate and age, and the $1 \sigma$ errors for the remaining quantities. The observed ("obs") and intrinsic ("int") magnitudes are also reported. The intrinsic stellar masses and SFRs are obtained by dividing the best-fit parameters resulting from the SED fit by $\mu$. It is worth noting that the values for ID14f include both components " $1+2$." The magnification $\mu$ is also reported and derived as described in the text.

\section{Discussion}

\subsection{The Nature of the Ionizing Source}

ID14 shows similarities with other star-forming systems identified at $z=3-7$ (Stark et al. 2015; Vanzella et al. 2016a; Mainali et al. 2016; Schmidt et al. 2017). We used the large grid of photoionization models described in Feltre et al. (2016) and Gutkin et al. (2016) to investigate the nature of the ionizing source. In all the diagnostic diagrams, ID14 belongs to the regions powered by star formation (e.g., see Figure 8). The main reason for that is the faintness of the He II $\lambda 1640$ line when compared to C IV $\lambda 1548$ and 1550 , and O III] $\lambda 16611666$, which would suggest a drop of the spectrum at energies just above 4Ry (see Mainali et al. 2016). From the archival $300 \mathrm{ks}$ Chandra exposure (Ogrean et al. 2015), we derived $2 \sigma$ upper limits on the X-ray flux of $1.6 \times 10^{-16}$ and $8.3 \times$ $10^{-16} \mathrm{erg} \mathrm{s}^{-1} \mathrm{~cm}^{-2}$ in the soft $(0.5-2 \mathrm{keV})$ and hard $(2-7 \mathrm{keV})$ band, respectively, taking into account the strong background emission due to the intra-cluster gas. At the redshift of ID14, these limits correspond to luminosities of $1.3 \times 10^{43}$ and $6.1 \times 10^{43} \mathrm{erg} \mathrm{s}^{-1}$ in the soft and hard band, respectively. They cannot exclude the possibility that an absorbed Seyfertlike AGN could be present.

In addition to the above limits and the line ratios, the fact that components " 1 " and " 2 " are spatially resolved and show very narrow nebular emission lines further supports the evidence that the source of ionizing radiation is dominated by stellar emission. This is also reminiscent of recent studies in the local universe (e.g., Annibali et al. 2015; Kehrig et al. 2015; Smith et al. 2016), where young super star clusters show narrow, high-ionization nebular lines. Although a low-luminosity AGN cannot be completely excluded, the presence of sub-structures in ID14 and the aforementioned characteristics support the conclusion that the star formation is likely to be the main source of ionizing radiation.

\section{2. $A L=0.004 L^{\star}$ Super Star Clusters at $\mathrm{z}=3.2222$}

ID14 is made of two main star-forming regions ("1, 2") of $\simeq 30 \mathrm{pc}$ effective radius each, with de-lensed magnitudes $\simeq 30.6\left(L=0.004 \mathrm{~L}_{z=3}^{\star}\right)$, possibly showing additional fainter sub-components. The low stellar mass $\left(<10^{7} M_{\odot}\right)$, low metallicity, young ages $(\lesssim 100 \mathrm{Myr})$, and hot stars are also distinctive features of this system.

The double-knot morphology of ID14 suggests two super star clusters of several $10^{6} M_{\odot}$ are present, and possibly composed of a mixture of stellar populations. In fact, the highionization (e.g., He II $\lambda 1640$ and C IV $\lambda 1548,1550)$ lines require a young $(\lesssim 10 \mathrm{Myr})$ stellar component, characterized by blackbody effective temperatures higher than 50,000 K (Steidel et al. 2014; Stark et al. 2015). The ages inferred from the SED fitting also support the presence of an underlying older $(\lesssim 100$ Myr) population.

The inferred specific star-formation rate (sSFR) is large $\left(>20 \mathrm{Gyr}^{-1}\right.$ ), a value comparable to those inferred by Stark et al. (2014) and Karman et al. (2017), suggesting that the system is still in a starburst phase and rapidly growing. Conservatively, adopting the lowest stellar mass $(2.5 \times$ $\left.10^{6} M_{\odot}\right)$ and the largest effective radius $\left(R_{C}=50 \mathrm{pc}\right)$ of the $68 \%$ intervals derived above, the observed stellar mass density of the star-clusters is relatively large, $>150 M_{\odot} \mathrm{pc}^{-2}$ (see also, Vanzella et al. 2017b).

\subsection{Deficient Lyo Emission in IDI4}

From the $\mathrm{H} \beta$ line flux, an estimate of the theoretical Ly $\alpha$ emission can be computed as $f(\operatorname{Ly} \alpha)=8.7 \times f(\mathrm{H} \alpha) \times$ $10^{1.048 \times E(B-V)}$, where we assume a case B recombination theory, $f(\mathrm{H} \alpha) / f(\mathrm{H} \beta)=2.7$ (Brocklehurst 1971). Adopting $E(B-V)=0.0(0.2)$ (for the nebular emission), the expected intrinsic Ly $\alpha$ flux is $1.7(2.7) \times 10^{-16} \mathrm{erg} \mathrm{s}^{-1}$, i.e., more than 150 times brighter than what was observed, and more than 12 times brighter than C IV $\lambda 1548,1550$. Despite that, the observed ratio ( $\mathrm{C} \operatorname{IV} \lambda 1548$ and $1550 / \mathrm{Ly} \alpha$ ) is $\gtrsim 15$. Therefore, $\operatorname{Ly} \alpha$ emission is strongly deficient. A conversion of the intrinsic Ly $\alpha$ emission into absorption can, in principle, be achieved by dust suppression of Ly $\alpha$ photons, geometrical effects leading to the scattering of Ly $\alpha$ photons out of the line of sight, or a combination of both. Similar physical conditions observed in local low-metallicity star-forming dwarf galaxies might help to better understand the nature of ID14 (though at different scales). For example, the low-metallicity, star-forming dwarf galaxy IZw 18 shows Ly $\alpha$ absorption, despite its low metallicity and young age. Also, galaxies of the LARS sample (or: local, $\mathrm{H} \alpha$ selected galaxies) with low velocity dispersion show a reduced Ly $\alpha$ escape (Herenz et al. 2016). A large number of scatterings in static, high column density gas can lead to an efficient suppression of $\operatorname{Ly} \alpha$ photons by even small amounts of dust. The Ly $\alpha$ absorption could also be caused by diffusion of the photons out of the observer direction (not by dust destruction). As a result, Ly $\alpha$ absorption can be observed also in dust-free galaxies (e.g., Atek et al. 2009). Verhamme et al. (2012) show directional variation in the Ly $\alpha$ escape from a simulated disk galaxy (see also Zheng \& Wallace 2014). This directional variation is reduced significantly in the presence of outflows (e.g., Duval et al. 2016) and/or for non-disk geometries, including modified shell models (Behrens et al. 2014) and/or clumpy ISM models (Gronke \& Dijkstra 2014), where the Ly $\alpha$ escape fraction varies by only a factor of order unity and thus do not provide the variations required to explain the observed flux ratio. However, the theoretical studies have considered systems with $\sim \mathrm{kpc}$ extents, and ignored observational aperture effects. The anisotropic 

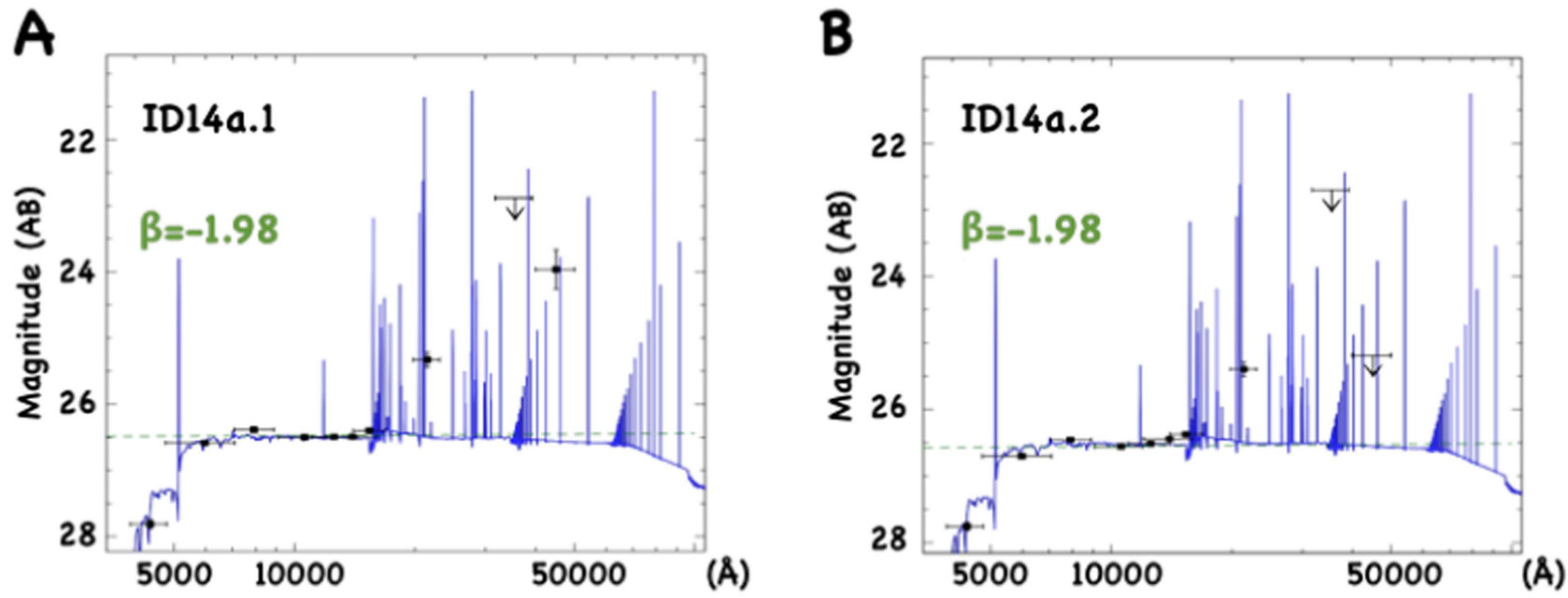

Figure 7. Best-fit SED solutions for components 1 and 2 of the ID14a image. The inferred physical quantities are reported in Table 2 . The greed dashed line is the ultraviolet slope $\beta$. The photometric discontinuity at $22000 \AA$ corresponds to the K-band, and is fully consistent with the measured flux of the optical emission lines observed with X-Shooter (see text)

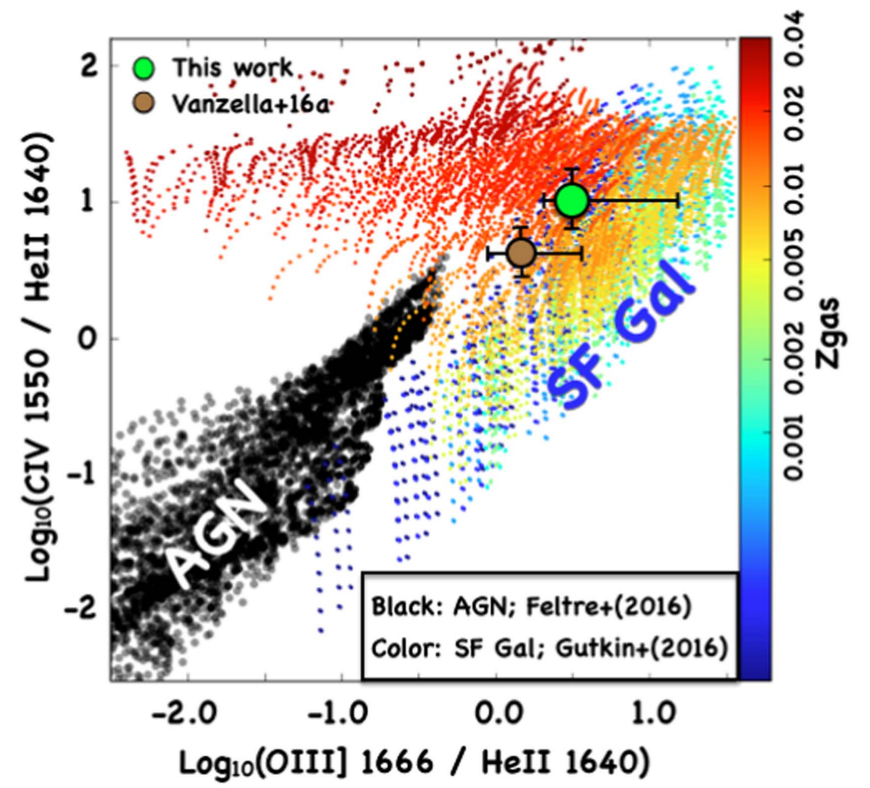

Figure 8. Diagnostic diagram separating AGN and star-forming powered sources with superimposed object ID14 and a similar object described in Vanzella et al. (2016a). Predictions are from photoionization models of Feltre et al. (2016; AGNs, gray-filled circles) and Gutkin et al. (2016). Star-forming galaxies are color-coded according to their gas phase metallicity, $Z_{\mathrm{gas}}$, as defined in Gutkin et al. (2016).

escape of Ly $\alpha$ is strongest if the source is "shielded" by a gas cloud of size similar to or larger than the emitting region (in this case, a few tens of parsecs), and weakened by photons scattering back into the line of sight. Therefore, having a very compact source as in this work is likely to enhance the directional variations, but further studies are needed to explore this possibility. Observational aperture effects are not an issue in the present case, given the wide MUSE FOV. The strong lensing effect can, in principle, modulate the spatial appearance of the Ly $\alpha$ emission. However, in the present case, no $\operatorname{Ly} \alpha$ emission is observed on top of the stellar ultraviolet continuum of images ID14a, b and c. Plausibly, ID14 would appear as a Ly $\alpha$-emitter if observed from a different view-angle, and would require a variation of the $\operatorname{Ly} \alpha$ intensity of two orders of magnitudes. One possibility is that even a small amount of dust extinction in a region enshrouded by neutral gas (or a partially neutral IGM) may explain the strong depression of the Ly $\alpha$ line. It is also worth noting that the CIV $\lambda 1548$ and 1550 positions measured with X-Shooter do not show clear signatures of outflowing gas (at velocities $>50 \mathrm{~km} \mathrm{~s}^{-1}$, see also Vanzella et al. 2016a), further supporting an efficient Ly $\alpha$ attenuation.

\subsection{A Spatially Offset Ultra-faint Ly $\alpha$ Emission}

As discussed in the previous section, ID14 is a system with prominent high-ionization lines that have energetic photons reaching at least $4 \mathrm{Ry}$. The deficiency of the Ly $\alpha$ emission at the spatial position where the metal lines and the stellar continuum arise suggests an efficient attenuation by dust and/or gas. However, such a process could be not so efficient in other directions. In the case of a non-uniform covering factor of the neutral gas, we may expect to recover the diffused Ly $\alpha$ photons in other directions and/or observe spatially offset fluorescent Ly $\alpha$ emission (Furlanetto et al. 2005; Weidinger et al. 2005). Indeed, we detected an offset and spatially extended Ly $\alpha$ emission $(\mathrm{S} / \mathrm{N}=7.4)$ at the same redshift as ID14 (separated by $\simeq 2.1 \mathrm{kpc})$ that may corroborate this possibility. The observed Ly $\alpha$ emission lies across the critical line produced by the galaxy cluster at the given redshift. The position of the critical line (the highest magnification) is strongly supported by the confirmed images of ID14, "image1" and "image2" (Figure 1, panel (B)). This also means the Ly $\alpha$ is strongly magnified and consequently highly spatially distorted (see Figure 4, magenta ellipse). The observed emitting region $\left(6.7 \mathrm{arcsec}^{2}\right)$ corresponds to de-lensed $7.6 \mathrm{kpc}^{2}$ and a de-lensed integrated flux of $1.7 \times 10^{-19} \mathrm{erg} \mathrm{s}^{-1} \mathrm{~cm}^{-2}$ if we adopt a median magnification $\mu=50$ (calculated within the same region). However, the real physical size and shape of the emission is affected by large uncertainties, especially where the magnifications formally diverge. This spatially offset Ly $\alpha$ suggests the possible presence of a neutral gas cloud at a distance of $2.1 \mathrm{kpc}$ from ID14, reached by the ionizing photons escaped from the system, where they can produce Ly $\alpha$ fluorescence (Behrens et al. 2014; Mas-Ribas \& Dijkstra 2016). The emerging spectral line 


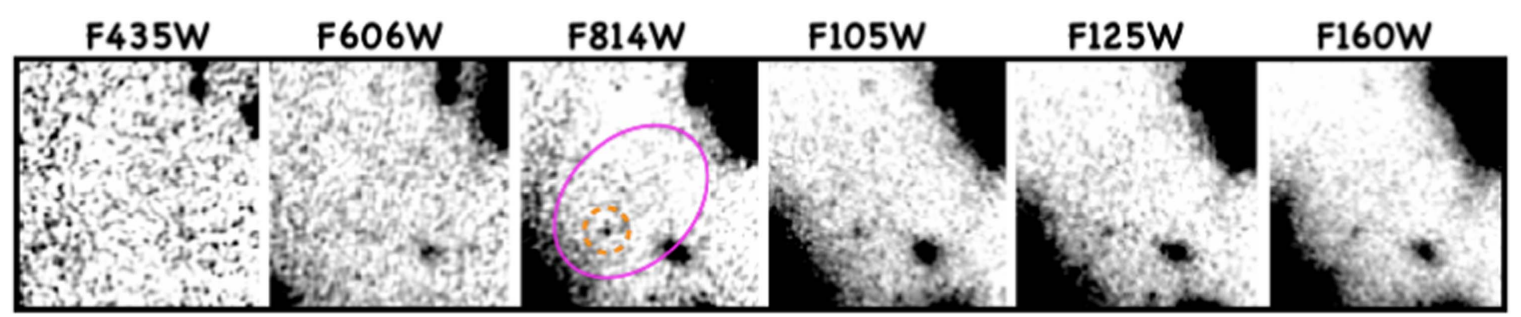

Figure 9. HST/ACS and WFC3 cutouts (1". 5 wide), centered on the diffuse Ly $\alpha$ emission marked with the magenta ellipse. The dashed-orange circle marks the position of the faint $m \sim 30$ (or $m>34$ de-lensed) underlying object if placed at the same redshift of the Ly $\alpha$ emission.

profile, apparently symmetric and narrow $\left(<40 \mathrm{~km} \mathrm{~s}^{-1}\right)$, would also support the fluorescence scenario. It is worth noting that ID14 shows optical oxygen line EWs and ratios similar to the ones of the Lyman continuum emitter discovered at $z=3.2$, named Ion 2 (with confirmed $>50 \%$ escaping ionizing radiation, see de Barros et al. 2016; Vanzella et al. 2016b) ${ }^{19}$ and some local extreme Green Peas sources (also Lyman continuum leakers, Izotov et al. 2016; Schaerer et al. 2016). A possible link between the large O32 index and the optically thin interstellar medium to ionizing radiation was explored with photo-ionization models by Jaskot \& Oey (2013). The large value observed here $(\mathrm{O} 32>10)$ may indicate a densitybounded condition (due to a deficient [O II] $\lambda 3727,3729$, Nakajima \& Ouchi 2014). This could be the case along some directions (not necessarily along the line of sight) in which the opacity to ionizing photons is low. Interestingly, similar transverse cavities possibly optically thin in the Lyman continuum have been observed in the local super star clusters, where expanding shells of gas (superbubbles) and blow-out regions can be produced by substantial mechanical feedback generated by massive super star clusters (e.g., Mrk71, James et al. 2016; Micheva et al. 2017).

In this scenario, the spatially offset fluorescent Ly $\alpha$ nebula might reside into an elongated cavity directly connecting it with ID14. We would also expect a large escape fraction of $\mathrm{Ly} \alpha$ photons along the same transverse direction (Behrens et al. 2014).

Alternatively, such Ly $\alpha$ emission might be produced by additional faint/undetected sources. We identified a faint object behind the diffuse Ly $\alpha$ emission (see the orange-dashed circle in Figure 4, panel (C)), detected in the HST bands with an average observed magnitude of $\simeq 30$. The faintness of the object prevents us from deriving any reliable photometric redshift or physical properties. We only note from Figure 9 an apparent drop in the F435W and F606W bands, compared to the other redder bands. If this object (or another undetected source) is responsible for the entire Ly $\alpha$ diffuse emission, then its de-lensed magnitude would be $\gtrsim 34(\mu \gtrsim 50)$ with an $\mathrm{EW}(\operatorname{Ly} \alpha)>450 \AA$ rest-frame (where the magnification is the median value calculated within $1^{\prime \prime} \times 1^{\prime \prime}$ centered on the object, orange circle in Figure 9). This would correspond to an absolute magnitude of $M_{U V}=-11$, and the very large Ly $\alpha$ EW would imply the presence of unusual stellar populations (e.g., Schaerer 2003; Dijkstra \& Wyithe 2007). Because the redshift of this object is unconstrained and/or we do not know if other undetected sources were present, more specific analysis will require deeper imaging with future instrumentation, such as JWST or ELT.

\footnotetext{
19 Although more massive $\left(<10^{9} M_{\odot}\right)$ and larger $(\simeq 300$ pc effective radius $)$, Ion 2 shows an EW of the $[\mathrm{O}$ III] $] \lambda 5007$ line $>1000 \AA$ rest-frame and a large $\mathrm{O} 32$ index $(>10)$; where the $\mathrm{O} 32$ index is $([\mathrm{O}$ III $] \lambda \lambda 4959,5007) /([\mathrm{O}$ II $] \lambda 3727,3729)$.
}

\section{Conclusions}

We have presented a strongly lensed pair of super starclusters at $z=3.2222$ separated by $\sim 300 \mathrm{pc}$, with magnitudes $m \simeq 30.6$ and effective radius of 30pc each. Both show highionization lines like C IV $\lambda 1548,1550$, He II $\lambda 1640$, O III] $\lambda 1661$, and 1666, as well as C III] $\lambda \lambda 1907$ and 1909 , with $\mathrm{S} / \mathrm{N}$ ratios ranging between 2 and 20, in addition to $\mathrm{H} \beta$, [O III] $\lambda \lambda \lambda 4959$, and 5007 optical lines with $\mathrm{S} / \mathrm{N}=4-31$, all of them showing narrow velocity dispersions, $\sigma_{v}<50 \mathrm{~km} \mathrm{~s}^{-1}$ (derived from MUSE), or $\sigma_{v} \lesssim 20 \mathrm{~km} \mathrm{~s}^{-1}$ (derived from X-Shooter). The presence of high-ionization lines (like He II $\lambda 1640$ ) and the physical properties inferred from the observed colors (SEDfitting) suggest that young stars are present $(<10 \mathrm{Myr})$, as well as a relatively evolved stellar component $(<100 \mathrm{Myr})$. A moderate dust attenuation $(E(B-V) \simeq 0.06)$ is also consistent with the observed colors. The inferred stellar masses are of a few $10^{6} M_{\odot}$ with a metallicity $1 / 10$ solar.

The main results can be summarized as follows:

1. Despite the fact that the above properties are often observed in Ly $\alpha$ emitting galaxies such as Green Peas, (e.g., Henry et al. 2015), ID14 does not show any Ly $\alpha$ emission aligned with the detected stellar continuum and ultraviolet metal lines, in all the observed multiple images ID14a, b, and c. In particular, the ratio C IV $\lambda 1548$ and $1550 /$ Ly $\alpha \gtrsim 15$ is uncommon, and it is the first case confirmed at high redshift and very low-luminosity regimes, as discussed here. A relatively low-velocity outflow, the presence of a screen of neutral gas and an (even moderate) amount of dust are all ingredients that may explain the Ly $\alpha$ attenuation.

2. A spatially offset and strongly magnified Ly $\alpha$ emission is detected at $\sim 2 \mathrm{kpc}$ transverse from ID14. There are two possible explanations: (A) an induced fluorescence in a nearby neutral gas cloud through a local and transverse escape of ionizing radiation from ID14 (this would also imply a transverse escape of $\operatorname{Ly} \alpha$ photons along the same route; see, e.g., Behrens et al. 2014) and/or (B) an in situ star-formation activity of an object barely (or not) detected in the deep HST images, with de-lensed $M_{U V} \simeq-11(m>34)$ and $\operatorname{EW}(\operatorname{Ly} \alpha)>450 \AA$ restframe. The latter, however, would imply unusual stellar populations (e.g., Dijkstra \& Wyithe 2007; Cantalupo et al. 2012).

The double lens effect (see Section 2), in which the observed flux ratios among the multiple images allow us to derive a relatively small error in a high-magnification regime, $\mu=40 \pm 7$, offers the opportunity to anticipate the expected future ELT capabilities, in terms of the spatial resolution and magnitudes, in the not-lensed fields (see also, Christensen et al. 2012; Vanzella et al. 2016a). In 
particular, apart from the detected pair of super star clusters (components " 1 " and " 2 "), additional sub-structures have been identified that possibly represent single H II regions and/or candidate proto-globular clusters (Vanzella et al. 2017b). More investigation is needed to fully characterize these systems. The spatial resolution (3-7 mas) achievable with the ELT instrumentation in conjunction with strong lensing (as in this case) will offer an opportunity to spatially resolve details of $1-2 \mathrm{pc}$ at $z>3$. It is also worth reporting what integration time would be needed with MUSE to achieve the same $\mathrm{S} / \mathrm{N}$ ratios of $\mathrm{UV}$ metal lines for a ID14-like not-lensed object. The most prominent among them is the C IV $\lambda 1548$ with de-lensed flux of $2.2 \times 10^{-19} \mathrm{erg} \mathrm{s}^{-1} \mathrm{~cm}^{-2}$ that would require $\mathrm{a} \simeq 1000 \mathrm{hr}$ integration time to reproduce the same $\mathrm{S} / \mathrm{N}=17$ reported here (or $\simeq 100 \mathrm{hr}$ for a $\mathrm{S} / \mathrm{N}=3$, with typical observing conditions and adopting the currently available ESO/MUSE exposure time calculator). Again, the optical spectra reported in this work offer a first glance at the future ELT-like spectra of similar objects in the non-lensed fields that are attainable with several hours of integration time.

Finally, we demonstrated (at least for this faint system) that the Ly $\alpha / \mathrm{C}$ IV ratio often reported in literature (e.g., Stark et al. 2014, 2015; Mainali et al. 2016) or any line ratio that includes the Ly $\alpha$ flux (e.g., Figure 7 in Shibuya et al. 2017) can be affected by a large line-of-sight variation of the Ly $\alpha$ visibility in low-luminosity regimes as well, therefore weakening its use as a diagnostic feature (e.g., Smit et al. 2017).

We thank the referee for useful comments that improved the manuscript. We thank K. Schmidt, L.I. Mas-Ribas, and R. Amorin for useful discussion. E.V. and S.C. acknowledge financial support from PRIN-MIUR 201278X4FL, Evolution of Cosmic Baryons: Astrophysical Effects and Growth of Cosmic Structures. C.G. acknowledges support by VILLUM FONDEN Young Investigator Programme through grant no. 10123. K.C. acknowledges funding from the European Research Council through the award of the Consolidator Grant ID 681627BUILDUP. L.C. is supported by Grant DFF 4090-00079. Based on observations collected at the European Southern Observatory for Astronomical research in the Southern Hemisphere under ESO programmes P095.A-0653, P094.A-0115 (B) and ID 094. A-0525(A). M.N., M.M., A.M., and P.R. acknowledge financial support from PRIN-INAF 2014 1.05.01.94.02.

\section{References}

Alavi, A., Siana, B., Richard, J., et al. 2016, arXiv:1606.00469 Amorín, R., Fontana, A., Pérez-Montero, E., et al. 2017, NatAs, 1, 0052 Amorín, R., Pérez-Montero, E., Contini, T., et al. 2015, A\&A, 578, A105 Annibali, F., Tosi, M., Pasquali, A., et al. 2015, AJ, 150, 143 Atek, H., Schaerer, D., \& Kunth, D. 2009, A\&A, 502, 791 Bacon, R., Accardo, M., Adjali, L., et al. 2010, Proc. SPIE, 7735, 773508 Behrens, C., Dijkstra, M., \& Niemeyer, J. C. 2014, A\&A, 563, A77 Bouwens, R. J., Illingworth, G. D., Oesch, P. A., et al. 2016, arXiv:1608.00966 Brocklehurst, M. 1971, MNRAS, 153, 471

Bruzual, G., \& Charlot, S. 2003, MNRAS, 344, 1000
Caminha, G. B., Grillo, C., Rosati, P., et al. 2016a, A\&A, 587, A80 Caminha, G. B., Grillo, C., Rosati, P., et al. 2016b, arXiv:1607.03462 Caminha, G. B., Karman, W., Rosati, P., et al. 2016c, A\&A, 595, A100 Cantalupo, S., Lilly, S. J., \& Haehnelt, M. G. 2012, MNRAS, 425, 1992

Cardamone, C., Schawinski, K., Sarzi, M., et al. 2009, MNRAS, 399, 1191 Castellano, M., Amorín, R., Merlin, E., et al. 2016, A\&A, 590, A31 Christensen, L., Laursen, P., Richard, J., et al. 2012, MNRAS, 427, 1973 de Barros, S., Vanzella, E., Amorín, R., et al. 2016, A\&A, 585, A51 Dijkstra, M., \& Wyithe, J. S. B. 2007, MNRAS, 379, 1589

Duval, F., Östlin, G., Hayes, M., et al. 2016, A\&A, 587, A77

Erb, D. K., Pettini, M., Steidel, C. C., et al. 2016, ApJ, 830, 52 Erb, D. K., Steidel, C. C., Trainor, R. F., et al. 2014, ApJ, 795, 33 Feltre, A., Charlot, S., \& Gutkin, J. 2016, MNRAS, 456, 3354

Fosbury, R. A. E., Villar-Martı́n, M., Humphrey, A., et al. 2003, ApJ, 596, 797

Furlanetto, S. R., Schaye, J., Springel, V., \& Hernquist, L. 2005, ApJ, 622, 7 Grillo, C., Karman, W., Suyu, S. H., et al. 2016, ApJ, 822, 78

Gronke, M., \& Dijkstra, M. 2014, MNRAS, 444, 1095

Gutkin, J., Charlot, S., \& Bruzual, G. 2016, MNRAS, 462, 1757

Henry, A., Scarlata, C., Martin, C. L., \& Erb, D. 2015, ApJ, 809, 19

Herenz, E. C., Gruyters, P., Orlitova, I., et al. 2016, A\&A, 587, A78

Holden, B. P., Oesch, P. A., González, V. G., et al. 2016, ApJ, 820, 73

Izotov, Y. I., Schaerer, D., Thuan, T. X., et al. 2016, MNRAS, 461, 3683

James, B. L., Auger, M., Aloisi, A., Calzetti, D., \& Kewley, L. 2016, ApJ, 816,40

Jaskot, A. E., \& Oey, M. S. 2013, ApJ, 766, 91

Karman, W., Caputi, K. I., Caminha, G. B., et al. 2017, A\&A, 599, 28

Kehrig, C., Vílchez, J. M., Pérez-Montero, E., et al. 2015, ApJL, 801, L28

Koekemoer, A. M., Avila, R. J., Hammer, D., et al. 2014, BAAS, 223, 254.02

Lotz, J., Mountain, M., Grogin, N. A., et al. 2014, BAAS, 223, 254.01

Lotz, J. M., Koekemoer, A., Coe, D., et al. 2016, arXiv:1605.06567

Mainali, R., Kollmeier, J. A., Stark, D. P., et al. 2016, arXiv:1611.07125

Mas-Ribas, L., \& Dijkstra, M. 2016, ApJ, 822, 84

Meneghetti, M., Natarajan, P., Coe, D., et al. 2016, arXiv:1606.04548

Merlin, E., Amorín, R., Castellano, M., et al. 2016, A\&A, 590, A30

Micheva, G., Oey, M. S., Jaskot, A. E., \& James, B. L. 2017, arXiv:1704. 01678

Nakajima, K., \& Ouchi, M. 2014, MNRAS, 442, 900

Ogrean, G. A., van Weeren, R. J., Jones, C., et al. 2015, ApJ, 812, 153

Peng, C. Y., Ho, L. C., Impey, C. D., \& Rix, H.-W. 2010, AJ, 139, 2097

Pérez-Montero, E., \& Amorín, R. 2017, MNRAS, 467, 1287P

Pilyugin, L. S., Vílchez, J. M., \& Thuan, T. X. 2006, MNRAS, 370, 1928

Rauch, M., Becker, G. D., \& Haehnelt, M. G. 2016, MNRAS, 455, 3991

Rhoads, J. E., Malhotra, S., Richardson, M. L. A., et al. 2014, ApJ, 780, 20

Schaerer, D. 2003, A\&A, 397, 527

Schaerer, D., Izotov, Y. I., Verhamme, A., et al. 2016, A\&A, 591, L8 Schmidt, K. B., Huang, K.-H., Treu, T., et al. 2017, arXiv:1702.04731 Shibuya, T., Ouchi, M., Harikane, Y., et al. 2017, arXiv:1705.00733 Smit, R., Swinbank, A. M., Massey, R., et al. 2017, MNRAS, 467, 3306S Smith, L. J., Crowther, P. A., Calzetti, D., \& Sidoli, F. 2016, ApJ, 823, 38 Stark, D. P., Ellis, R. S., Charlot, S., et al. 2017, MNRAS, 464, 469 Stark, D. P., Richard, J., Siana, B., et al. 2014, MNRAS, 445, 3200 Stark, D. P., Walth, G., Charlot, S., et al. 2015, MNRAS, 454, 1393 Steidel, C. C., Rudie, G. C., Strom, A. L., et al. 2014, ApJ, 795, 165 Vanzella, E., Balestra, I., Gronke, M., et al. 2017a, MNRAS, 465, 3803 Vanzella, E., Calura, F., Meneghetti, M., et al. 2017b, MNRAS, 467, 4304 Vanzella, E., De Barros, S., Cupani, G., et al. 2016a, ApJL, 821, L27 Vanzella, E., de Barros, S., Vasei, K., et al. 2016b, ApJ, 825, 41 Vanzella, E., Fontana, A., Zitrin, A., et al. 2014, ApJL, 783, L12 Verhamme, A., Dubois, Y., Blaizot, J., et al. 2012, A\&A, 546, A111 Weidinger, M., Møller, P., Fynbo, J. P. U., \& Thomsen, B. 2005, A\&A, 436,825

Zheng, Z., \& Wallace, J. 2014, ApJ, 794, 116

Zitrin, A., Meneghetti, M., Umetsu, K., et al. 2013, ApJL, 762, L30 